\title{
The Economic Determinants of Ethnic Assimilation
}

\author{
By \\ Carmel U. Chiswick \\ Department of Economics \\ University of Illinois at Chicago \\ and \\ IZA, Bonn, Germany \\ June 2007
}

\begin{abstract}
$\underline{\text { Abstract }}$
A human capital model is developed that distinguishes between ethnic-specific skills (applicable only to a specific indigenous or immigrant group) and shared or general skills. An important determinant of assimilation is the extent to which these two forms of human capital are complements, thus promoting both assimilation and ethnic persistence, or anti-complements, promoting either assimilation or ethnic retention but not both. Implications of the model are developed for various applications including intermarriage, the effects of group size, language and religion as a basis for ethnic mergers, and the transfer society as a potential barrier to assimilation.
\end{abstract}

JEL Classifications - J11, J15, J24, Z13

Keywords - Ethnicity, Human Capital, Ethnic Intermarriage, Transfer Society, Education

\section{Mailing address:}

Professor Carmel Ullman Chiswick

Department of Economics (m/c 144)

University of Illinois at Chicago

601 S. Morgan Street

Chicago 60607-7121
Phone: (312) 996-8721

FAX: (312) 996-3344

e-mail: cchis@uic.edu 


\section{THE ECONOMIC DETERMINANTS OF ETHNIC ASSIMILATION \\ by \\ Carmel U. Chiswick ${ }^{1}$}

\section{Introduction}

The relevance of ethnicity for economic analysis derives from the fact that ethnic groups differ in their consumption patterns and in their supply of resources to the economy at large. Some of these differences are relatively minor, accounting for less variation in supply and demand than individual differences in tastes and preferences. Others have effects that can be pronounced and persistent on markets and on the institutional basis of the economy. By exploring the economic determinants of ethnic assimilation, this paper also develops implications for the persistence of differences among ethnic groups and some implications of these differences for society as a whole.

Ethnic group members develop consumption patterns within the community specific to the group and not shared by other members of the larger society. Ethnicityspecific goods and services (referred to as "ethnic goods") may include food and clothing items, religion, music, or ethnic newspapers, radio, and TV stations (Chiswick and Miller 2005). Human capital related to these consumption patterns may also be ethnic-specific. Important examples of ethnic human capital are skills related to ethnic languages and religions, as well as gender and age roles. Much of this human capital is formed within

\footnotetext{
${ }^{1}$ Department of Economics, University of Illinois at Chicago. This paper has evolved over several years during which it has benefited from interactions with many people. An earlier version was presented at the Second Annual Migrant Ethnicity Meeting (IZA) and at the 2006 Annual Meeting of the European Society for Population Economics (ESPE), and a much earlier version at the Twelfth International History Congress, all of which were sources of helpful suggestions. The author is especially grateful to Barry R. Chiswick, Alan Olmstead, Timothy Hatton, and Guillermina Jasso for their encouragement and comments. The author, however, takes full responsibility for the contents of this paper.
} 
the family and community, often fairly early in life, and affects the way in which people relate to the larger society in which the ethnic group is embedded. The process of ethnic human capital formation (referred to here as "ethnic education") may itself be groupspecific, a possibility that will be explored later in this paper.

Ethnic differences in consumption and labor supply also induce differences in patterns of investment in general human capital, resulting in cases of occupational "specialization" within ethnic groups and to dramatic differences in schooling levels between "successful" and "disadvantaged" ethnic groups (B. Chiswick 1988; Borjas 1992; Borjas 1995; Lehrer 2004). These in turn influence ethnic differences relating to more intimate matters such as health and medical care, marriage patterns, fertility rates and family life. To a large extent, it is these differences that give an ethnic group visibility and contribute to its image within the larger society.

The analysis developed in this paper begins with the assumption that each individual belongs unambiguously to one and only one ethnic group. ${ }^{2}$ For simplicity, there are only two distinctive ethnicities within a larger society that they both share. ${ }^{3}$ Individuals face tradeoffs between goods that are shared (i.e., demanded by persons of both ethnicities) and those that are specific to their own ethnic groups. Similarly, they allocate their education budgets between shared and ethnic-specific human capital. Part

\footnotetext{
${ }^{2}$ In some usage, an ethnic group is defined residually after controlling for such characteristics as race, religion, or place of origin. This study uses the term broadly to include groups defined by these characteristics as well. For simplicity, these characteristics are assumed to be unambiguous and well known, so that every member of the population knows the ethnicity of every other member.

${ }^{3}$ Individuals who distance themselves from ethnic particularism may view themselves as having no ethnicity. Typically, however, people who share this viewpoint develop their own social structures and thus create the equivalent of a more or less new ethnicity. The fact that these people have origins in different ethnic groups is not relevant for the distinction between ethnic-specific and shared types of human capital.
} 
II develops this model and uses it to distinguish between two concepts of ethnic assimilation. Part III applies the model to various issues related to assimilation, including inter-ethnic marriage patterns, group size effects, and ethnic conflict. Part IV concludes with a discussion of implications for social and economic policy aimed at fostering the assimilation of ethnic groups.

\section{An Economic Model of Ethnic Identity}

Membership in an ethnic group is understood to be a "good" in the sense that it is desirable but not costless. That is, identification with the group provides benefits both tangible and intangible, but requires diversion of scarce resources from other uses. Although ethnicity can not be purchased directly, it is best thought of as a z-good produced by combining ethnic goods and services (e.g., ethnic clothing, food, entertainment, charities, club memberships) and time expenditures on group-specific activities. ${ }^{4}$ Each ethnic group is part of the larger society and each has a group-specific ethnic z-good, E, that effectively defines its identity.

\section{A. The Ethnic Consumer}

Utility-maximizing consumers allocate their time between ethnic and general activities. The problem can be expressed as:

$$
\begin{aligned}
& \text { (1) } \begin{aligned}
\operatorname{Max} \mathrm{U}(\mathrm{E}, \mathrm{Y}) \quad \text { subject to } \quad \mathrm{L}_{\mathrm{E}}+\mathrm{L}_{\mathrm{Y}}+\mathrm{L}_{\mathrm{S}}=\mathrm{L}^{*} \\
\text { where } \mathrm{E}=\text { Ethnicity } \\
\mathrm{Y}=\text { All other (shared) goods and services } \\
\mathrm{L}_{\mathrm{E}}=\text { Time spent in ethnic-specific activities } \\
\mathrm{L}_{\mathrm{Y}}=\text { Time spent in general activities } \\
\mathrm{L}_{\mathrm{S}}=\text { Total time spent in human capital formation }
\end{aligned}
\end{aligned}
$$

\footnotetext{
${ }^{4}$ For a discussion of ethnic goods see (Chiswick and Miller 2005)
} 
and $\mathrm{L}^{*}$ is the total time available for all purposes.

Human capital may or may not be work-related, referring rather more generally to the skills and experiences relevant for producing the consumption goods, E and Y. It is useful to distinguish between ethnic human capital, the skills and experiences specific to E production thus useful only for members of that ethnic group, and shared human capital, the skills and experiences that raise productivity for Y production and are thus useful to members of all groups. For example, ethnic human capital, $\mathrm{H}_{\mathrm{E}}$, might include a group-specific ethnic language, religion, or customs affecting family relationships, while human capital shared by all ethnic groups, $\mathrm{H}_{\mathrm{Y}}$, would include the common language and labor market skills useful for everyone without regard to ethnicity.

Ignoring for simplicity the role of purchased goods and services, let the two consumption goods, $\mathrm{Y}$ and $\mathrm{E}$, be self-produced with human capital specific to each activity:

$$
\begin{aligned}
& \text { (2) } \mathrm{E}=\mathrm{g}\left(\mathrm{h}_{\mathrm{E}} \mathrm{L}_{\mathrm{E}}\right) \\
& \text { (3) } \mathrm{Y}=\mathrm{f}\left(\mathrm{h}_{\mathrm{Y}} \mathrm{L}_{\mathrm{Y}}\right) \\
& \text { where } \mathrm{h}_{\mathrm{E}}=\text { level (quality) of ethnic human capital } \\
& \text { and } \mathrm{h}_{\mathrm{Y}}=\text { level (quality) of general human capital. }
\end{aligned}
$$

In this specification the total amount of human capital, $\mathrm{H}_{\mathrm{E}} \equiv \mathrm{h}_{\mathrm{E}} \mathrm{L}_{\mathrm{E}}$ or $\mathrm{H}_{\mathrm{Y}} \equiv \mathrm{h}_{\mathrm{Y}} \mathrm{L}_{\mathrm{Y}}$ respectively, is the sole input for producing the corresponding consumption good. Each ethnic group is thus characterized by its own group-specific human capital.

In the present analysis, the term "ethnic education" refers to any investment in ethnic-specific human capital, a skill-formation process that enhances the productivity of resources deployed within the group (i.e., for making the ethnic z-good) but not 
productivity in the general labor market or in general consumption activities. Ethnic education in this sense begins early in childhood with ethnic-specific parenting styles and family customs, later expanding to include socialization within the ethnic community and more or less formal training in group-specific skills. The rate of return to ethnic education depends not only on individual preferences (a lifestyle choice) but also on the production function for ethnic experience.

Each type of human capital is the output of an educational process with its own production function, the main input to which is the student's time. These can be written inversely as cost functions, expressing the time cost of education as a function of the level of skill to be acquired.

(4) $\mathrm{L}_{\mathrm{S}}=\mathrm{L}_{\mathrm{YS}}+\mathrm{L}_{\mathrm{ES}}$

(5) $\mathrm{L}_{\mathrm{YS}}=\varphi\left(\mathrm{h}_{\mathrm{Y}}\right) / \alpha \quad \varphi^{\prime}, \varphi^{\prime \prime}>0$

(6) $\mathrm{L}_{\mathrm{ES}}=\left[\gamma\left(\mathrm{h}_{\mathrm{E}}\right)-\omega \mathrm{h}_{\mathrm{Y}} \mathrm{h}_{\mathrm{E}}\right] / \alpha \quad \gamma^{\prime}, \gamma^{\prime \prime}>0$

where $\mathrm{L}_{\mathrm{YS}}=$ Time spent in general (shared) learning activities and $\mathrm{L}_{\mathrm{ES}}=$ Time spent in ethnic-specific learning activities.

The efficiency parameter $\alpha>0$ in equations (5) and (6) is an individual-specific positive constant that capture differences in learning abilities, with $\alpha>1$ for a high ability student (who can acquire a given level of skill in less time) and $\alpha<1$ for a low-ability student. The constant parameter $\omega$ is group-specific and indicates the degree to which the acquisition of general human capital imposes an external effect on ethnicity-specific education. For example, if $\omega>0$ a greater level of general human capital $\left(h_{Y}\right)$ would make it less costly to acquire any given level of ethnic education $\left(\mathrm{h}_{\mathrm{E}}\right)$, while if $\omega<0$ the opposite 
would be true. ${ }^{5}$

The consumer's problem is solved by maximizing the Lagrangian function:

$$
£=\mathrm{U}\left(g\left(\mathrm{~h}_{\mathrm{E}} \mathrm{L}_{\mathrm{E}}\right), f\left(\mathrm{~h}_{\mathrm{Y}} \mathrm{L}_{\mathrm{Y}}\right)\right)-\lambda\left\{\mathrm{L}_{\mathrm{E}}+\mathrm{L}_{\mathrm{Y}}++\left[\gamma\left(\mathrm{h}_{\mathrm{E}}\right)+\varphi\left(\mathrm{h}_{\mathrm{Y}}\right)-\omega \mathrm{h}_{\mathrm{Y}} \mathrm{h}_{\mathrm{E}}\right] / \alpha-\mathrm{L}^{*}\right\}
$$

where equations (4)-(6) have been substituted into the time constraint to eliminate the schooling time variables. The first-order conditions can be solved to yield

$$
\begin{array}{ll}
\text { (8) } U_{g} g^{\prime} h_{E}=U_{f} f^{\prime} h_{Y} \\
\text { (9) } L_{E} / h_{E}=\left[\gamma^{\prime}-\omega h_{Y}\right] / \alpha \\
\text { (10) } L_{Y} / h_{Y}=\left[\varphi^{\prime}-\omega h_{E}\right] / \alpha \\
\text { (11) } L^{*}=L_{E}+L_{Y}+\left[\gamma\left(\mathrm{h}_{\mathrm{E}}\right)+\varphi\left(\mathrm{h}_{\mathrm{Y}}\right)-\omega \mathrm{h}_{\mathrm{Y}} \mathrm{h}_{\mathrm{E}}\right] / \alpha
\end{array}
$$

Equaton (8) equates the marginal rate of substition in consumption between ethnicspecific and common (shared) uses of time to -1 , the slope of the time budget line, requiring that the marginal value of time be the same in both consumption activities. Equations (9) and (10) equate the slopes of the human capital quantity-quality isoquants, $\mathrm{L}_{\mathrm{E}} / \mathrm{h}_{\mathrm{E}}$ and $\mathrm{L}_{\mathrm{Y}} / \mathrm{h}_{\mathrm{Y}}$ respectively, to the marginal cost of the corresponding type of education. These conditions allocate time to each type of education up to the point where its marginal value in human capital formation is the same as the opportunity cost of that time in consumption activities. Equation (11) restates the overall time constraint for all activites.

Solving equations (9) and (10) for $h_{E}$ and $h_{Y}$, respectively, substituting the result into equations (8) and rearranging terms yields:

\footnotetext{
${ }^{5}$ A similar externality with respect to the general education process is ignored here for the sake of simplicity. If the term $\omega^{*} \mathrm{~h}_{\mathrm{Y}} \mathrm{h}_{\mathrm{E}} / \alpha$ were to be subtracted from the right-hand side of equation (5), the only effect on equation (7) would be to replace the constant parameter $\omega$ with $\left(\omega^{*} \omega^{*}\right)$. The following equations would have to be adjusted accordingly, but there would be no substantive difference in their interpretation.
} 


$$
\text { (12) } \frac{U_{g} g^{\prime} L_{E}}{U_{f} f^{\prime} L_{Y}}=\left[\frac{\gamma^{\prime}-\omega h_{Y}}{\varphi^{\prime}-\omega h_{E}}\right]
$$

The expression on the left-hand side of equation (12) is the marginal rate of substitution in consumption between $h_{E}$ and $h_{Y}$, the slope of an indifference curve between levels of the two types of education. The right-hand side is the slope of a production possibility frontier $(\mathrm{PPF})$ between $\mathrm{h}_{\mathrm{E}}$ and $\mathrm{h}_{\mathrm{Y}}$.

The PPF itself is one of a family of curves defined by $L_{S}=\left[\gamma+\varphi-\omega h_{Y} h_{E}\right] / \alpha$, each corresponding to a fixed level of resources - i.e., to a fixed value of $L_{S}-$ devoted to general and ethnic training combined. The amount human capital attainable with a given amount of resources is positively related to the parameter $\alpha$, being greater for people of high ability with $\alpha>1$ and lower for people with $\alpha<1$. If the amount of time devoted to education is allowed to vary with $\alpha$, equation (12) implies an expansion path with a positive slope (for both $h_{Y}$ and $h_{E}$ normal). For any given combination of $h_{Y}$ and $h_{E}$, however, the slope of the PPF is independent of $\alpha$ and thus the same for all members of the group.

Equations (9) and (10) may also be solved for $\mathrm{L}_{\mathrm{E}}$ and $\mathrm{L}_{\mathrm{Y}}$, respectively, the results substituted into equation (11), and the terms rearranged to obtain:

$$
\text { (13) } L^{*}=\left[h_{E} \gamma^{\prime}+h_{Y} \varphi^{\prime}+\gamma+\varphi-3 \omega h_{E} h_{Y}\right] / \alpha
$$

Thus the education production functions can be used to convert the overall time constraint into an expression that is solely a function of skill levels, $h_{E}$ and $h_{Y}$, the ability parameter $\alpha$, and the externality parameter $\omega$. Like the PPF, this time-constrained opportunity set is larger for high-ability individuals, but its slope is independent of $\alpha$ and 
thus the same for all group members. ${ }^{6}$

The overall solution to the consumer's problem occurs at the point where the individual's expansion path crosses the time constraint of equation (13) associated with the appropriate value of $\alpha$. This point uniquely determines the optimal combination $h_{E}$ and $h_{Y}$, whose values may then be substituted into equations (9) and (10) to obtain the optimal allocation of consumption time and into equations (5) and (6) to obtain the optimal time devoted to each type of education. Although consumers focus their attention on the time allocation problem, the focus in this paper will be on the educational outcomes uniquely associated with consumers' decisions.

The key parameter in the model developed here is $\omega$, an indicator of cultural tension between the ethnic group and the shared environment. When $\omega$ is positive the two kinds of human capital are acquired by means of complementary learning processes: the greater the level of $h_{E}$ embodied in a person the more efficiently he can learn general skills, and the greater the level of shared human capital, the more efficiently he can acquire ethnic-specific skills. The opposite is true when $\omega$ is negative, indicating that high levels of ethnic human capital (hence attachment to the ethnic communmity) make it more difficult to acquire general skills and vice versa. The parameter $\omega$ will thus differ across ethnic groups to a degree that depends on the relationship between each group's culture relative to the shared culture of the larger society.

Figure 1 illustrates the consumer's decision and its sensitivity to the groupspecific parameter $\omega$. The indifference map between ethnic-specific skills (on the

\footnotetext{
${ }^{6}$ A sufficient (but not necessary) condition for this slope to be negative is $\omega \leq 0$, meaning that the external effects of general education on ethnic education (and vice versa) are non-positive.
} 
horizontal axis) and shared skills (on the vertical axis) has been drawn to represent an individual with a strong preference for the ethnic good. ${ }^{7}$ Three possible production possibility frontiers have been drawn, all using the same total resources and thus with endpoints at A and B. The PPF corresponding to a group where ethnic education and general education are mutually independent (i.e., where $\omega=0$ ) has been emphasized with a dark curve. In this case the optimal combination of human capital would be at point $\mathrm{C}$, and the expansion path is indicated by a similarly dark line. ${ }^{8}$

For purposes of comparison, Figure 1 shows how different PPF curves would affect outcomes for the same individual with a strong preference for ethnic consumption. The PPF corresponding to positive externalities in the education process $(\omega>0)$ is tangent to the indifference map at point $\mathrm{D}$, on a higher indifference curve than $\mathrm{C}$ and characterized by greater investments in both types of human capital but with relatively more $h_{Y}$ than in the mutually-independent case. In contrast, the optimal investment if there are negative externalities between the two educational processes $(\omega<0)$ is at point $\mathrm{E}$, on a lower indifference curve and with greater specialization in ethnic human capital. Although not shown on this graph, the expansion path passing through $\mathrm{E}$ is to the right and less steep than the path through $\mathrm{C}$, while the one passing through $\mathrm{D}$ is to its left and more steep.

\footnotetext{
${ }^{7}$ For an interesting empirical exercise in measuring these axes for people in Germany see (Constant et al. 2006). A promising approach to measurement elsewhere is suggested in (Jasso et al. 2006)

${ }^{8}$ The expansion path connects the tangencies between the indifference map and a family of PPF curves for which $\omega=0$, only one of which is shown in Figure 1.
} 


\section{B. The Ethnic Group}

By definition, an ethnic group is the set of all consumers who consume its ethnicspecific good E, and all consumers in a given ethnic group face the same family of PPF curves and time constraints. Individual consumers vary in their tastes and preferences for the ethnic good, however, as well as in the efficiency with which they acquire new human capital. Thus each person's human capital expansion path is individually determined, as is the optimal point on that path. To analyze the implications of these individual decisions for the ethnic community as a group and for the larger society in which it is embedded, each member of the group is assumed to make a decision based on a common family of PPF curves but an expansion path determined by his or her own indifference map.

Figure 2a illustrates the case of an ethnic community with positive $\omega$, indicating that the two learning processes are mutually complementary so that its PPF curves will be bowed outward (i.e., its convexity will be high), and Figure $2 \mathrm{~b}$ illustrates the case of negative externalities where $\omega$ is negative and large in magnitude. In both graphs the dotted-line expansion path corresponds to the indifference map of persons with a strong preference for the ethnic good, shown in Figure 1 as having a tangency to a PPF at points D (for Figure 2a) or E (for Figure $2 b$ ). The solid-line expansion path corresponds to the indifference map of people with weak preferences for the ethnic good or strong preferences for the consumption basket shared by all groups. Those with more balanced preferences will have expansion paths somewhere between these two extremes.

People locate themselves along their respective expansion paths according to their educational abilities. In Figure 2, a shaded oval area around each of the two extreme 
expansion paths is intended to suggest a scatter of outcomes for group members with similar (but not identical) preferences. Not indicated in the graph is the scatter of outcomes for people in between these two extremes. Figure 2a illustrates the case of a group with very high value of $\omega$, where extreme differences in preferences nevertheless yield similar outcomes so the in-between area is small and the group is fairly homogeneous. Figure $2 \mathrm{~b}$ illustrates the case where $\omega$ is very low (highly negative), where a balanced human capital portfolio is fairly costly. People with balanced preferences would have outcomes within this region but the tendency would be for the scatter of outcomes to thin out as it approaches the center and to concentrate at lower levels of human capital. The scatter of points for groups with moderate values of $\omega$ would have more members in the area between thes two extremes.

\section{Ethnic Identity and Assimilation}

The concept of ethnic assimilation is socially determined and depends importantly on the shared culture of the larger society. Two extreme cases will be considered here, referred to briefly as "pluralism" and "homogeneity," respectively. As its name suggests, a pluralistic society is one in which multiple ethnic groups coexist within a larger social framework that they all share. In this context, an ethnic group is considered to be assimilated whenever its members accept the values, attitudes and norms of the shared culture and participate more or less actively in its economic and social life. In contrast, a homogeneous society is one where everyone is expected to conform to a shared ideal, typically associated with the ethnic culture of one or more specific groups. In this 
context, a group is not viewed as assimilated until its members adopt the dominant ethnicity and the minority group effectively disappears.

\section{Acculturation in a Pluralistic Society}

Groups in a pluralistic society assimilate with respect to each other when they share values, goals, and activities, giving their members an incentive to invest in a common set of human capital attributes. That is, the greater the level of shared human capital embodied in a person, $\mathrm{h}_{\mathrm{Y}}$, the more he or she has in common with members of other ethnic groups and hence, by definition, the greater the degree of acculturation. ${ }^{9}$ For simplicity, suppose there is some threshold level of shared human capital, $\mathrm{h}_{\mathrm{Y}}^{*}$, beyond which an individual may be characterized as acculturated. By the same token, a group may be characterized as assimilated into the shared culture of a society if a large proportion of its members are acculturated because they embody at least $\mathrm{h}_{\mathrm{Y}} *$ amount of shared human capital.

Figures $2 \mathrm{a}$ and $2 \mathrm{~b}$ illustrate this threshold with a horizontal line at $\mathrm{h}_{\mathrm{Y}}{ }^{*}$. The portion of the shaded ovals above this line represent people who are assimilated in this sense, sharing the values, attitudes, experiences and knowledge common to all ethnic groups in the larger society. The portion below this line represent the unacculturated, people who do not share much human capital with the mainstream society and are thus perceived, by themselves and by others, as outsiders.

By this definition assimilation need not mean homogeneity, for only the amount of shared human capital, $h_{\mathrm{Y}}$, is relevant for determining participation in a shared culture. The amount of ethnic-specific human capital, $\mathrm{h}_{\mathrm{E}}$, is an important determinant of his or her

\footnotetext{
${ }^{9}$ Note that this definition applies symmetrically to all groups, including those described as "dominant" because of their large size or cultural influence.
} 
ethnic identity and hence participation in an ethnic community, but it does not affect the extent to which an individual participates in the larger society.

Again for simplicity, suppose there is some threshold level of ethnic human capital, $\mathrm{h}_{\mathrm{E}}{ }^{*}$, required for a person to have a clear association with a particular ethnic group. Persons with more than this amount of $h_{E}$ identify with the ethnic community and would lose the value of their ethnic human capital if they were to exit. Those with less than $h_{E} *$ have little attachment to the community and would incur little cost if they were to leave. While members of the latter group are sometimes described as "assimilated" because of their low ethnic identity, they may or may not be active participants in the society's shared culture.

The relationship between ethnic identity and assimilation is best seen by considering both definitions simultaneously, as illustrated in Figure 3 by the division into four quadrants. The axes and the shaded ovals duplicate those in Figures $2 \mathrm{a}$ and $2 \mathrm{~b}$, respectively. Individuals whose education choices places them to the right of the vertical line at $\mathrm{h}_{\mathrm{E}}{ }^{*}$, in quadrants I or II, have strong ethnic identities, while those in quadrants III or IV, to the left of this line, do not. The people in quadrants I and IV embody more shared human capital than $h_{Y} *$ and are thus assimilated in the sense that they share the same values, attitudes, experiences and knowledge as the members of other ethnic groups in the larger society. Those in quadrants II and III are below the threshold value of $\mathrm{h}_{\mathrm{Y}}$ * and are in this sense "outside" the shared culture. People in quadrant II are "outsiders" because they identify with an ethnic group that does not share the society's common values. Those in quadrant III constitute a marginalized underclass that participates in neither the common culture nor an ethnic subculture. 
The taxonomy suggested in Constant et al. (2006) makes a distinction between "assimilation" and the "integration" of ethnic groups into the mainstream of society. ${ }^{10}$ People in quadrant I, with high levels of general human capital even though their consumption patterns continue to include ethnic goods and hence a demand for ethnicspecific human capital, are said to be "integrated" into the larger society. Together they form an "integrated" ethnic community, in contrast to the people in quadrant II who constitute a "separated" ethnic community. The term "assimilated" is reserved for the people in quadrant IV who have little ethnic human capital, and hence low productivity in ethnicity-specific consumption patterns, but otherwise participate in the general society. ${ }^{11}$ In this respect they differ from the "marginalized" people in quadrant III who are at best loosely attached to society at large and to their own ethnic group.

People in quadrant IV are assimilated, and those in quadrant II are unassimilated (separatist), with respect to any society, whether pluralistic or homogeneous. As Figure $3 b$ suggests, these quadrants dominate in groups for which ethnic education is most anticomplementary with respect to general education, inducing people to specialize in either ethnic or shared human capital. Those who choose the ethnic-specific investment path

\footnotetext{
${ }^{10}$ Constant et al. (2006) focuses on measuring the degree of ethnic assimilation rather than an analysis of its determinants. Its classification of ethnic groups for this purpose is much the same as the four quadrants illustrated in Figure 3 of the present paper.

${ }^{11}$ In common parlance, the term "assimilation" often combines both concepts. For example, American Jewry in the early $20^{\text {th }}$ century was an immigrant community that viewed assimilation as a highly desirable goal, but in the late $20^{\text {th }}$ century was more likely to view it as a serious problem. The earlier community understood assimilation as a movement from quadrant II into quadrant I, investing heavily in $h_{Y}$ often at the expense of investments in $\mathrm{h}_{\mathrm{E}}$. The later generation was centered in quadrant I but included many people in quadrant IV whose connections to the Jewish community were weak or ambivalent. While both communities used the term "assimilation," the earlier period used it in its broader sense (quadrants I and IV) while the narrower usage (quadrant IV) is implied during the later period.
} 
have low levels of $h_{Y}$ and thus tend to be socially - and perhaps also geographically separated from the mainstream. Group members who choose the shared-human-capital investment path tend to fall in quadrant IV, where low levels of ethnic human capital reduce the priority placed on ethnic lifestyles and high levels of shared human capital provide a common bond with persons of other ethnicities whose priorities are similar.

Figure 3a illustrates the propensity to assimilate for groups in which ethnicspecific and general educations are mutually complementary. This complementarity in education raises the incentive to invest in both kinds of human capital, inducing outcomes in which most members are likely to be in quadrant I and a less-educated minority in quadrant III. The group would thus be dominated by productive and culturally assimilated members of the larger society who also have a strong ethnic identity and are likely to participate in the ethnic community. Members who assimilate in the sense of reduced ethnic identity would tend to have low levels of shared human capital and hence would also find it difficult to participate fully in the larger society.

\section{Assimilation in a Homogeneous Society}

In a society whose shared culture emphasizes the benefits of homogeneity, ethnic particularism is discouraged and assimilation is defined as a decision to identify with the mainstream "shared" society. The decision to switch ethnicity in order to join the mainstream, whether informally or through some sort of formal conversion process, requires that the benefits of switching outweigh the cost. In this respect it is analogous to migration: the higher the stock of non-transferable ethnic skills, the higher the cost of switching and greater the gain required to compensate for the loss associated with changing groups. 
If the largest cost of joining the mainstream is determined by the loss of nontransferable ethnic human capital, the vertical line at $h_{E} *$ in Figure 3 can be interpreted as the borderline dividing the ethnic group between leavers and stayers. People with human capital endowments to the right of this line have high levels of ethnic attachment and high costs of leaving the group, while those to the left of this line have low levels of ethnic human capital and hence a low cost of exit from the group. ${ }^{12}$

Figure 3a suggests that members of successful ethnic groups with high positive values of $\omega$ are unlikely to assimilate in this sense, and that members of those groups who choose to switch affiliation are likely to be the least educated within the community. In contrast, for groups with large negative values of $\omega$, illustrated by Figure $3 b$, the ethnic community will be divided into those that choose to assimilate by joining the mainstream (in the vertical oval mostly to the left of $h_{E}{ }^{*}$ ) and strongly-identified ethnics who resist assimilation (in the horizontal oval that is mostly below $\mathrm{h}_{\mathrm{Y}}{ }^{*}$ ). In a society that places a high value on ethnic homogeneity, high levels of group-specific human capital associated with religion, language, or family life that reinforce clear group boundaries may thus serve as a de facto barrier to assimilation even if the ethnic group itself does not actively discourage its members from switching.

\section{Applications and Extensions}

The model developed above can be applied to a variety of issues associated with the assimilation of ethnic groups with respect to the larger society or with respect to each

\footnotetext{
${ }^{12}$ The rate of return to changing one's ethnic identity is the net present value of the gain in utility divided by the net present value of the cost of switching. If everyone faces the same prospects of gain and the same cost function for acquiring human capital specific to the new ethnicity, all differences in rates of return arise from individual differences in learning ability, $\alpha$, and in the amount of ethnic human capital $h_{E}$ specific to the origin group.
} 
other. In doing so, it is often necessary to relax one or more of the restrictions assumed in the previous section for the sake of simplicity. This section considers four such issues. Section A discusses the relationship between ethnic intermarriage and assimilation. Section B discusses the effects of ethnic group size on assimilation. Section C considers the possible endogeneity effect that can occur when two or more minority ethnic groups merge with each other, and Section D considers the conflict that arises when ethnic groups jockey for economic or political power within the larger society. In each case, the basic human capital model is applied with appropriate modifications (if any).

\section{A. Assimilation by Ethnic Intermarriage}

Low levels of ethnic human capital would facilitate movement by individuals across ethnic boundaries, whether purposeful (by conversion or intermarriage) or inadvertently as a byproduct of other consumption decisions. To the extent that such movements result in assimilated families with relatives who are ethnically identified, they may lead ipso facto to a blurring of the social boundaries by which ethnic groups are distinguished from the mainstream and from each other. Vague boundaries in turn facilitate further blending of ethnic identities as they reduce the cost of switching into the relatively homogeneous mainstream. To the extent that ethnic intermarriage occurs in such a society, it can be understood as both a cause and a symptom of ethnic assimilation.

Viewing ethnic intermarriage as symptomatic of assimilation requires the narrower definition (i.e., quadrant IV) most often associated with an assumption that the society has homogeneity as its ideal. In a pluralistic society, intermarriage may be associated with the merging of ethnic identities (as, for example, when people of English and Scottish descent become Anglo-Americans, or when people of Pakistani and 
Moroccan origin become Moslem-Americans) but it is generally irrelevant as an indicator of assimilation into the larger society. In a society that places a premium on homogeneity, however, intermarriage is closely associated with assimilation into the dominant mainstream group, with the weakening of ethnic ties, and with the eventual disappearance of the ethnic group. With this caveat in mind, the model developed above has implications for the economics of intermarriage as a vehicle for ethnic assimilation.

Marital compatibility is generally greatest for people with similar levels and types of human capital (Lehrer and Chiswick 1993; Lehrer 1996; Lehrer 1998). The optimal marital sort would thus tend to pair people who are close to each other with respect to both $h_{Y}$ and $h_{E}$. People who closely identify with an ethnic group because they have $h_{E}>$ $\mathrm{h}_{\mathrm{E}}{ }^{*}$ would prefer partners of the same ethnicity, those who are assimilated into the shared culture because they have $h_{Y}>h_{Y} *$ would prefer partners who are similarly acculturated, and those whose human capital falls below these thresholds are likely to marry each other.

To see the implications of this for intermarriage between members of different ethnic groups, Figure 4 illustrates the situation for the simplest case where two ethnicities coexist in the same society. ${ }^{13}$ The portions of Figures $4 \mathrm{a}$ and $4 \mathrm{~b}$ to the right of their respective origins, with horizontal axes that measure ethnic skills of Group A, are a duplicate of Figures $3 \mathrm{a}$ and $3 \mathrm{~b}$. The portions to the left of the origin measure the ethnic skills of Group B from right to left, but otherwise they also duplicate Figures 3a and 3b, respectively. While it is not necessary (or even likely) that two ethnic groups would have

\footnotetext{
${ }^{13}$ This analysis implicitly assumes that the content of ethnic education for the two groups is mutually compatible or at least neutral (i.e., does not generate hostility) with respect to each other.
} 
the same production functions for ethnic human capital, the present discussion is sufficiently general to permit abstraction from such differences.

Since people with high levels of ethnic human capital are most likely to seek marriage partners with similarly high levels of $h_{E}$, the propensity for intergroup marriage is low for people in quadrants I and II of either group. The propensity for ethnic intermarriage is greater for people in quadrants III and IV in part because they have low productivity in ethnic consumption and in part because their rate of return to switching groups is higher than that of people with more ethnic-specific human capital. It may even be that alienation from their respective ethnic communities provides a common bond for people on either side of the vertical axis but close to it, thus further increasing the likelihood of intermarriage.

Figure 4a illustrates the case where ethnic and general educations are mutually complementary, providing incentives to invest in both kinds of human capital. People with large investments in human capital would tend to have relatively high levels of ethnic as well as shared human capital and thus be less likely to seek partners outside the group. Ethnic intermarriage is more likely to occur among the less educated, who are also more likely to include persons with weak preferences for the ethnic good. At all levels of education, however, members with different preferences (and thus in different shaded ovals) have similar combinations of $h_{E}$ and $h_{Y}$ and thus can form stable marriages with each other.

In contrast, differences in preferences are extremely important for marital propensities in Figure 4b, where it is much less likely that people of the same ethnicity but with different preferences would match themselves with each other. When ethnic and 
general educations are anti-complementary (i.e., when each generates negative externalities for the other) people face reduced incentives to invest in human capital and tend toward specialization in one or the other type. In Figure $4 b$, the only people with a strong incentive to seek partners within the group are those with strong preferences for the ethnic good and heavy investments in ethnic human capital. In contrast, persons with weak preference for the ethnic good (or a strong preference for the shared good) are close to the vertical axis (most likely in quadrant IV) and thus unlikely to seek marital partners among those in quadrant II. People with low levels of $h_{E}$ may marry each other or they may match themselves with partners of a different ethnic origin but with similarly low levels of their respective ethnic human capital.

The configurations illustrated in Figures $4 \mathrm{a}$ and $4 \mathrm{~b}$ are symmetrical in that the general human capital is equally complementary with the ethnic human capital of the two groups. This need not be the case. For example, the human capital shared by everyone might be highly complementary to the ethnic human capital for Group A but anticomplementary to that of Group B. In this case the graph to the right of the vertical axis would look like the corresponding portion of Figure 4a and the graph to the left of the axis would look like Figure 4b. People in Group A with low ethnic attachment would also have low levels of $h_{Y}$, but people with low levels of attachment to Group B would have high levels of $h_{Y}$. Ethnic intermarriage is thus much less likely to occur between these groups even among people who are assimilated.

\section{B. Group Size Effects}

An important asymmetry occurs when the two ethnic groups differ in the size of their respective populations. Suppose the two ethnic groups in Figure 4 were identical in 
every respect except that Group A is ten times larger than Group B. Then each quadrant would have ten people in Group A for every person in Group B. In the extreme case where Group B experiences a 100 percent intermarriage rate, the intermarriage rate for Group A could never exceed 10 percent. Even if the propensity to intermarry were the same for both groups, in the sense illustrated by the symmetry in Figure 4, the probability that people in quadrants III and IV of one group could find a match from the other would be very high for members of Group B but very low for members of Group A. Thus the observed intermarriage rate would be much higher for group B than for group A, and would have very different implications for their respective ethnic communities.

Within a single ethnic group, the relative number of people within each set of preferences may also have implications for intermarriage rates. ${ }^{14}$ Suppose, for example, that 75 percent of the people in figure $4 \mathrm{~b}$ are in the high-ethnicity quadrant II and only 15 percent in quadrant IV, with the other 10 percent loosely scattered in the other two quadrants. Suppose further that the threshold level of education $h_{Y} *$ corresponds to graduation from high school. This group would be characterized as low-achieving because the large majority of its members have less than $h_{Y}{ }^{*}$. The "exceptional" group members who are high-school graduates would be found in quadrant IV and would be likely to marry outside the group not only because their ethnic identity is weak but also because their numbers are few.

Now suppose these numbers were reversed, with 15 percent of the people in the high-ethnicity, low-education quadrant II and 75 percent in the high-education, low-

\footnotetext{
${ }^{14}$ Size is much less important for a group illustrated by Figure $4 \mathrm{a}$, where people in different ovals may marry each other and where the "exceptional" members with high out-marriage rates would tend to be relatively few and among the least educated.
} 
ethnicity quadrant IV. Then the group might be perceived as assimilated, with an "exceptional" minority preserving the ethnic heritage. Persons in the disadvantaged minority sub-group can be expected to marry each other and so have a low out-marriage rate. Those in the assimilated majority might be open to ethnic intermarriage, but they also have a large pool of potential partners in the same ethnic subgroup. Ethnic outmarriage would be socially acceptable - even welcomed - within the assimilated higheducation subgroup, but its intermarriage rate is not predictable.

This simple example illustrates several hypotheses linking education levels and ethnic intermarriage rates. For ethnic groups characterized by high levels of general education and strong ethnic identity, out-marriage rates would tend to be higher among the less-educated minority. For groups with high levels of general education and weak ethnic identity, out-marriage rates would tend to be higher the smaller the size of the group. For groups with strong ethnic identity and low levels of general education, outmarriage rates would be higher among the well-educated minority.

The association between ethnic intermarriage rates and assimilation is thus observable only in certain special cases. It can be expected in a society that places a high value on ethnic homogeneity, where ethnicity-specific human capital is ipso facto anticomplementary to the shared human capital of the larger society. It can also be expected in a pluralistic society if the ethnic group is a small minority whose membership includes people who are marginally attached to the group. Assimilation is much less likely to be associated with inter-ethnic marriages for large groups in a pluralistic society or for groups that differ greatly in their investment in shared human capital. 
The size of an ethnic group also has an indirect effect on intermarriage rates because of economies of scale in the production and consumption of ethnic goods. Investment in language skills are clearly more productive the larger the pool of people speaking the same language, and the more people who speak an ethnic language the greater the incentive to express oneself in it and hence the more extensive the literature that emerges. Human capital formation in childhood is more effective if all members of the family share the same ethnicity, and the cost is further reduced when family life is embedded in an ethnic community with its larger stock of human capital and attendant social institutions. The utility-generating productivity of a group's ethnic human capital is thus amplified when there are many consumers with high levels of the human capital specific to the same ethnicity.

Since shared experiences and goals are the bases for many voluntary social organizations, ethnic groups often exhibit characteristics typical of membership societies or clubs. Of particular interest here is the mutual complementarity among members of various forms of participation. Each individual finds the utility-generating productivity of resources devoted to ethnic consumption to be enhanced by the active participation of other group members or, conversely, finds the benefits of participation to be lower in a group where other members are apathetic. This generates "bandwagon" effects that can be either positive or negative. ${ }^{15}$

\footnotetext{
${ }^{15}$ For some interesting applications of this phenomenon see Iannaccone (1992), Chiswick and Chiswick (2000), and Kahanec (2006).
} 


\section{Ethnic Mergers}

The assumption until now has been that each individual acquires human capital specific to one and only one ethnicity and that ethnic human capital has little betweengroup transferability. This assumption can now be relaxed. Even people who identify strongly with one ethnic group may learn about (or acquire human capital specific to) another. In societies with multiple ethnic groups, two groups may have some ethnic human capital in common (e.g., languge or religion) even though it is not shared by everyone in the larger society.

To illustrate the new possibilities, each of the two-dimensional graphs in Figure 4 can be folded $90^{\circ}$ along its vertical axis to make a third dimension, with one of its axes labeled Ethnic Skills Group A and the other labeled Ethnic Skills Group B. Individuals who specialize in the human capital of their own ethnic group would still be found near one of these axes (and hence near the ovals illustrated in Figure 4), but those who acquire some human capital specific to the other group would be represented by a point somewhere in this third dimension. In the extreme case, where the two types of ethnic human capital are mutually compatible (complementary) and people from both groups tend towards the central area in this plane, the groups may effectively assimilate with respect to each other and form a combined ethnicity.

In general, the greater the ethnic specificity of family-related human capital, the greater the incentive to choose a marriage partner from within the group (Chiswick and Lehrer 1991; Lehrer and Chiswick 1993). For ethnic groups that differ with respect to parenting skills and family relationships, intermarriage is most likely to occur among people who are not planning to have children or are otherwise distant from close 
relatives. If ethnic differences have little bearing on childrearing skills and family experiences, intermarriage is less difficult and thus more likely. Ethnic group boundaries are further blurred when marital partners from different groups raise children with ambiguous affiliations, whether they belong to both groups or to neither. Intermarriage is thus both a symptom and a cause of assimilation.

This suggests an important relationship between religion, language and ethnicity that tends to link the two aspects of group identity. Passing on an ethnic language and the formation of religious human capital during childhood and youth are important functions of the family and community. If these types of human capital are transferable across ethnic groups that share the same language and/or religion, an ethnic intermarriage would be less costly between them than between groups speaking a different language or practicing another religion. In practice, the linguistic barrier to intermarriage can be overcome since members of all ethnic groups share the common language of the larger society. ${ }^{16}$ The children of such intermarriages typically learn only the common language, with the long-run implication for ethnic languages to fade out. Finding a shared religion is more difficult, so ethnic intermarriages are less likely to cross religious boundaries; the long-run tendency would be for intermarriage to blur ethnic distinctions within religious groups so that ethnicity and religion become identified with each other.

\section{Ethnic Conflict}

When one ethnic group is "dominant," whether because of its relative size, its economic influence, or its political power (history), the distinction between general and

${ }^{16}$ This condition is less likely to be met by immigrants than by their native-born children, although immigrants in small immigrant communities have a stronger incentive to learn the language of the larger society and thus are more likely to intermarry. 
ethnic human capital for that group is not always clear. Its situation would be the one illustrated by Figure 3a, where the formation of general skills and ethnic skills are so highly correlated that for practical purposes the distinction between the two is blurred. Members of the dominant ethnicity would have a clear economic advantage over the members of other ethnic groups. There is also a tendency for the dominant group to be described as "mainstream" and for assimilation to be perceived as investment in its ethnicity-specific human capital.

Although this confusion is common in many countries, it generates a number of problems that may retard the assimilation of minority groups. For example, it increases the likelihood that a minority ethnic groups faces the kind of negative externalities illustrated by Figure 3b. Suppose, for example, that ethnic Germans are the dominant group in Germany and that members of this group invest heavily in knowledge about science (a general skill) and German customs regarding marriage and family life (ethnic skills). Scientists would be observed to follow these customs, and a spurious causal relationship might be inferred. This would make it more difficult for members of, say, a Turkish minority in Germany to invest in a science education, for they might think that by doing so they would be exchanging their Turkish family lifestyle for a German one, effectively switching from one ethnic group to another. Confounding the ethnic human capital of a dominant group with general human capital is thus a potential source of ethnic tension that may retard assimilation.

Economic agents have two basic strategies for expanding their opportunities for consumption, by raising productivity or by seeking to transfer to themselves (whether by taxation, fiat, theft or extortion) the rents earned by other agents (Anderson and Hill 
1989). In the "production" economy free markets maximize aggregate consumption and cause the income distribution to be Pareto optimal. In the "transfer" economy this is not the case, for even when transfers are limited to scarcity rents (in which case they would not change the level or allocation of existing resources) they represent a reduction in capital formation and hence a deadweight loss in aggregate production.

Any ethnic group with a high positive value of $\omega$ will have many members with high levels of both $h_{Y}$ and $h_{E}$ who are successful participants in production activities. Many different productive skills are encompassed in the concept of $h_{Y}$, and there is a tendency for group members to cluster in occupations for which their ethnic human capital is especially complementary. Even if they don't dominate the labor supply for a particular occupation, the advantage in skill-acquisition that attracts them implies a scarcity rent that tends to be higher than that of co-workers from different ethnicities.

In contrast when $\omega$ is negative, strongly-attached members of an ethnic group tend to be low-earning, isolated socially and perhaps also geographically from other ethnic groups with whom they share little human capital. The combination of isolation and disadvantage can foster a sense of grievance, but more importantly it raises the return to rent-seeking behavior relative to production activities. Ethnic differences in scarcity rents provide economic incentives to participate in the "transfer" economy, where laws or other arrangements are designed to benefit one ethnic group at the expense of others. The incentives to support such an economy are positively related to ethnic-group differences in the levels of economic rent and inversely related the cost of enforcing the transfer.

Ethnic groups differ in their attitudes toward rent seeking as an acceptable alternative to production, as well as in their power to enforce such exchanges, and they 
vary accordingly in their support for these strategies. A transfer can be ethnic-based either explicitly, as in affirmative-action programs, or implicitly, as in large-family subsidies whose benefits fall disproportionately on a few groups. Where such thnicbased transfer strategies are viable, they constitute a clear incentive for groups to resist assimilation into the shared mainstream (Epstein 2006). As with any other economic activity, the efficiency of rent seeking can be enhanced by an increase in the relevant skills. Unlike production skills, which are potentially shared by all groups, rent-seeking skills can be focused on group-specific goals. When this is the case, a relatively high investment in such group-specific transfer skills would provide an economic incentive to resist assimilation.

Although the notion of a "mainstream" or dominant ethnic group is fairly common, the specific group that defines the mainstream can change over time or from one region to the next even within a single country. Ethnic dominance in this context is endogenous, and jockeying for that status can be a source of ethnic conflict. The modern era has added a new dimension to the transfer society and hence a new economic impetus for ethnic conflict. Even as high productivity raises the stakes by increasing aggregate income, public-sector provision of services provides an institutional structure that facilitates extensive income transfers. Thus control of a national government is a prize worthy of considerable sacrifice, and many ethnic groups have chosen to divert resources to that end. This suggests that it is no coincidence that economic development in the nineteenth and twentieth century has been accompanied by the emergence of particularly powerful nationalist movements on the part of many ethnic groups for whom sovereignty was not previously an issue. 
Rent-seeking skills would be especially valuable in countries where transfers have been relatively more important than production as a source of income, whether because productivity is low (as in some LDCs) or because transfers are especially large (as in the former Soviet Union) or both. Thus it may be no coincidence that some of the most virulent ethnic violence of our time occurs in countries with low levels of modern human capital or a history of inefficiency in production. Economically induced ethnic violence may be self-limiting (if only because it dissipates the very rents that serve as its reward) ${ }^{17}$ Yet it can be very destructive and have immiserating long-run effects on the economy as a whole.

\section{Conclusion and Policy Implications}

The focus of this paper has been on understanding economic forces that support the assimilation of ethnic groups into a larger society. A microeconomic model of investment in ethnic human capital served as the basis for modeling the determinants of ethnic identity and the relationship between the ethnic group and the general society. This relationship provided a context for defining assimilation in two different ways, depending on whether the general society is pluralistic or homogeneous with respect to its ethnic ideals. The likelihood that an ethnic group would assimilate was shown to be sensitive to the degree to which investment in its ethnic human capital complements investments in general (shared) forms of human capital.

The degree of complementarity or anti-complementarity was shown to have important implications not only for persistence of the ethnic group but also for such

${ }^{17}$ The image of a "cash cow" - yielding a steady flow of income with no diminution of capital - meets the fable of the "goose that lays a golden egg" - a source of easy income only as long as it remains intact. 
ethnic-group differences in general investments as educational attainment and demographic behavior. The model was applied to an analysis of inter-ethnic marriages, often considered a symptom of assimilation. This association was shown to be important when the ethnic group is small relative to the general society, when investment in the group's ethnic human capital is anti-complementary to investments in general human capital, and when the general society has homogeneity as an ideal. Assimilation is less likely to associate with intermarriage for relatively large ethnic groups, for groups with a high degree of complementarity between investments in ethnic and general human capital, or for groups in a pluralistic society.

Some of the model's assumptions were relaxed in order to allow consideration of factors associated with the endogeneity of ethnic identification over time and space. In some cases, groups with ethnic human capital that is mutually complementary may merge to form a larger ethnic community that can benefit from economies of scale and positive bandwagon effects. For example, second- and third-generation immigrant groups with a common religion often merge to form a single ethnic identity based on religion rather than country of origin. In other cases, between-group differences in the nature of ethnic human capital may generate differences in general skills and hence in scarcity rents earned by group members. When these differences are large they provide an incentive for low-rent groups to extract income transfers from high-rent groups, a process that typically involves some degree of ethnic conflict and serves as a disincentive to assimilation.

The model developed in this paper suggests several different approaches to achieving assimilationist goals. First, and perhaps most importantly, it suggests that 
ethnic identity per se is neither undesirable nor a barrier to the assimilation of individuals into the larger society. In a pluralistic society the goal of assimilation is not to erode all ethnic distinctions, but rather to enhance the common culture and economic opportunities shared by all groups. Adaptations of the shared culture that increase its compatibility with a particular ethnic group would also have the effect of raising that group's externality parameter, $\omega$, and thus encourage its members to invest in the shared human capital that constitutes assimilation. Policies that welcome ethnic diversity within the larger society without encouraging separation would have this effect. A policy of multiculturalism would fit this description as long as it is genuinely inclusive, but multicultural policies that elevate some groups (e.g., "disadvantaged" relative to "advantaged") or that subsidize the acquisition of ethnic human capital relative to general human capital would not have the desired effect.

Within an ethnic group, any adaptations that raise positive externalities between ethnic-specific and general education, $\omega$, would have the effect of increasing assimilation without loss of ethnic identity (integration). If the production possibility frontier (PPF) for the acquisition of these two types of skill is concave to the origin, this will bow it out even further and thus have the effect of strengthening ethnic identity as well. If the externalities are negative and sufficiently large, cultural adaptations that make ethnic skills more compatible with the larger society (i.e., that make $\omega$ less negative) encourage more members to assimilate, but they also reduce the likelihood that assimilated members will leave the group. For such groups the challenge is to find adaptations to the ethnic culture that reduce negative externalities without sacrificing the ethnic identity that gives the group its special character. 
Apart from these changes in human capital, economic policies that encourage inter-ethnic rent-seeking rivalryies will retard assimilation and should be avoided. Any transfer society based on group differences would ipso facto generate such rivalries. Whether income transfers are implemented by a public tax-transfer system or by voluntary charity, a "disadvantaged" ethnic group with a large proportion of transfer recipients may develop institutions that implicitly support low levels of assimilation and encourage "disadvantaged" status. The challenge is to design transfers that are neutral with respect to group membership to avoid the unintended consequence of erecting barriers to assimilation. 


\section{References}

Anderson, T. and P. J. Hill (1989). The Birth of a Transfer Society. Lanham, MD, University Press of America.

Becker, G. S. (1981). A Treatise on the Family. Cambridge, Mass., Harvard University Press.

Borjas, G. J. (1992). "Ethnic Capital and Intergenerational Mobility." Quarterly Journal of Economics 107(1): 123-150.

Borjas, G. J. (1995). "Ethnicity, Neighborhoods, and Human Capital Externalities." American Economic Review 85: 365-390.

Chiswick, B. R. (1988). "Differences in Education and Earnings Across Racial and Ethnic Groups: Tastes, Discrimination, and Investments in Child Quality." Quarterly Journal of Economics 103: 571-597.

Chiswick, B. R. and C. U. Chiswick (2000). "The Cost of Living Jewishly and Jewish Continuity." Contemporary Jewry 21: 78-90.

Chiswick, B. R. and P. W. Miller (2005). "Do Enclaves Matter in Immigrant Adjustment?" City and Community 4(1): 5-35.

Chiswick, C. U. (2006). "An Economic Perspective on Religious Education: Complements and Substitutes in a Human Capital Portfolio." Research in Labor Economics 24: 449-467.

Chiswick, C. U. and E. L. Lehrer (1991). "Religious Intermarriage: An Economic Perspective." Contemporary Jewry 12: 21-34.

Constant, A., L. Gataullina and K. F. Zimmerman (2006). "Ethnosizing Immigrants". Discussion Paper, 30.

Epstein, G. S. (2006). "Migrants, Ethnicity and Employment". Unpublished manuscript, 29.

Iannaccone, L. R. (1992). "Sacrifice and Stigma: Reducing Free-riding in Cults, Communes, and Other Collectives." Journal of Political Economy 100(2): 271291.

Jasso, G., D. S. Massey, M. Rosenzweig and J. P. Smith (2006). "Interethnic Marriage, Citizenship, and Measurement of Ethnicity".

Kahanec, M. (2006). "Ethnic Specialization and Earnings Inequality: Why Being a Minority Hurts but Being a Big Minority Hurts More". Unpublished manuscript, 64.

Lehrer, E. L. (1996). "The Determinants of Marital Stability: A Comparative Analysis of First and Higher Order Marriages." Research in Population Economics 8: 91-121.

Lehrer, E. L. (1998). "Religious Intermarriage in the United States: Determinants and Trends." Social Science Research 27: 245-263.

Lehrer, E. L. (2004). "Religion as a Determinant of Economic and Demographic

Behavior in the United States." Population and Development Review 30: 707-726. 
Lehrer, E. L. and C. U. Chiswick (1993). "Religion as a Determinant of Marital Stability." Demography 30(3): 385-404.

Figure 1:

Strong Preference for Ethnic Goods

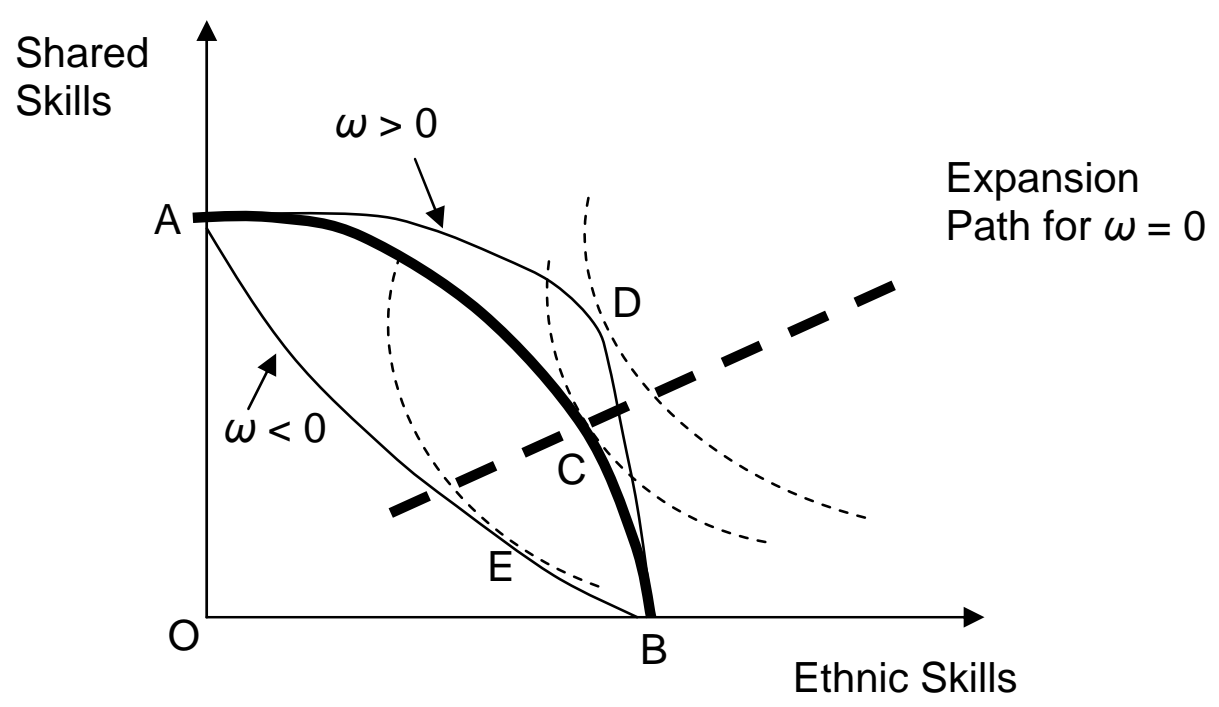


Figure 2a

Derived Demand for Ethnic Human Capital: $\omega>0$

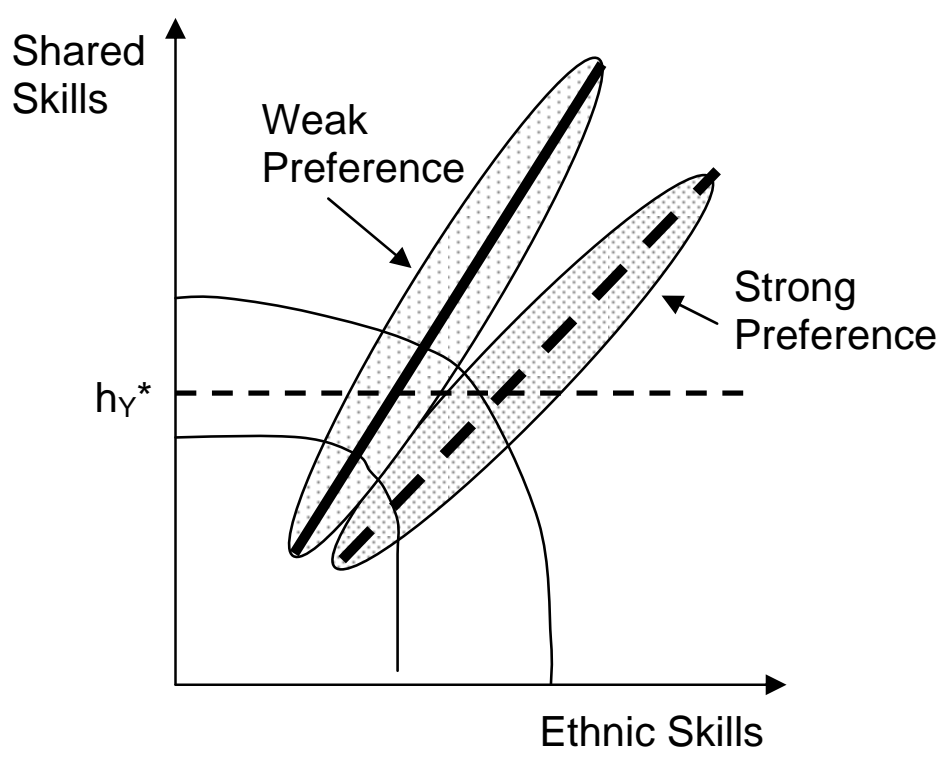


Figure $2 b$

Derived Demand for Ethnic Human Capital: $\omega<0$

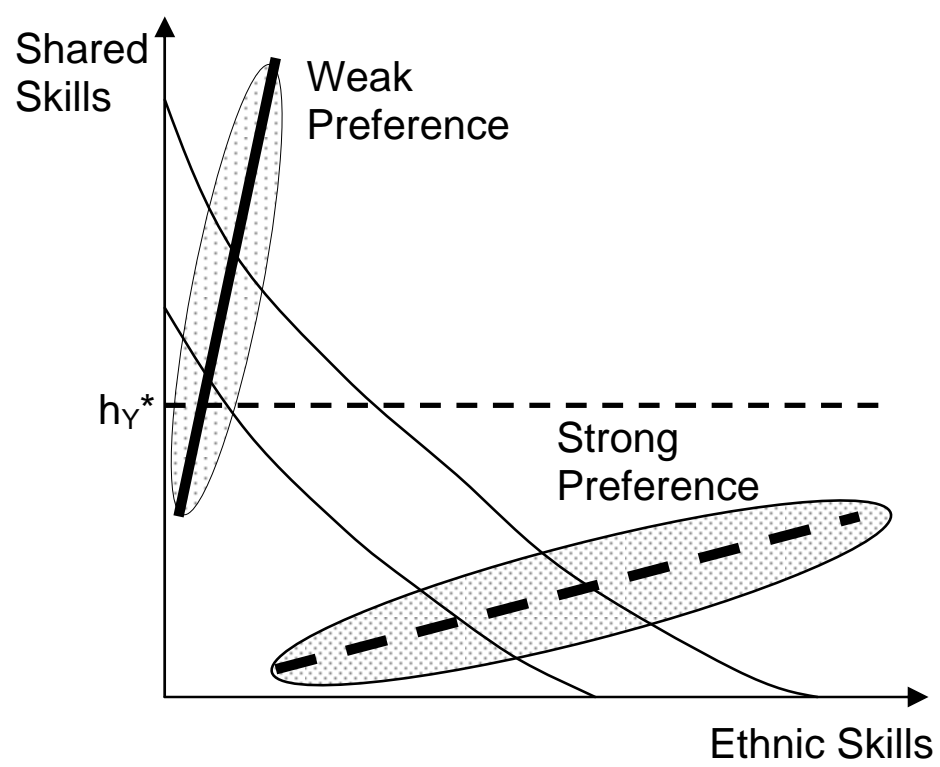


Figure 3a

Propensity to Assimilate: $\omega>0$

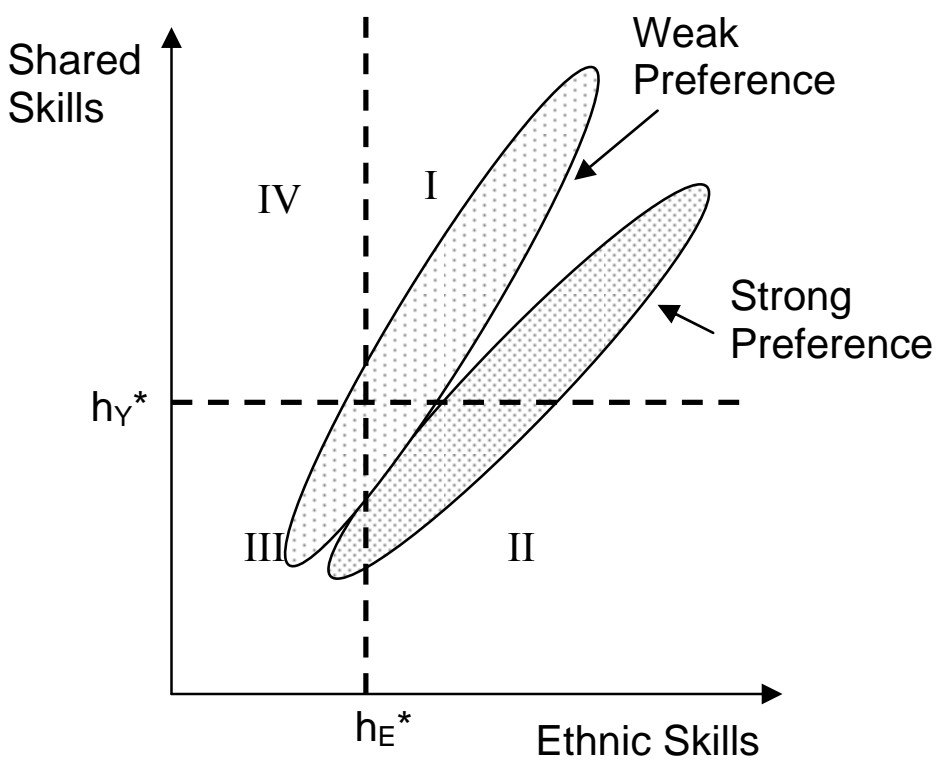

Figure $3 b$

Propensity to Assimilate: $\omega<0$

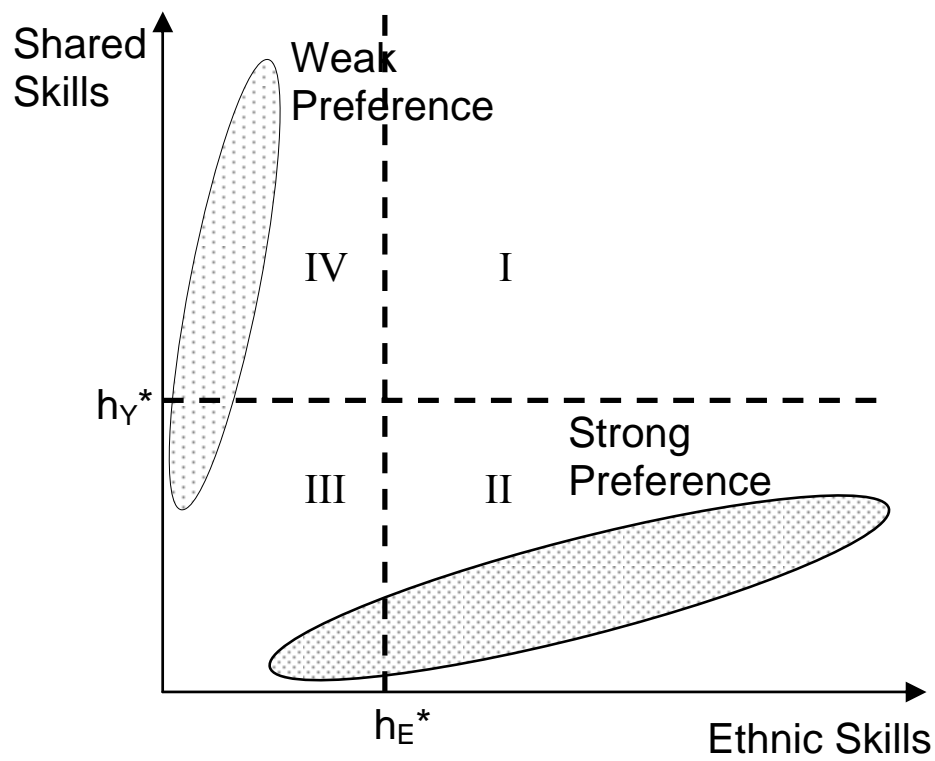


Figure $4 \mathrm{a}$

Two Ethnic Groups with Positive Externalities $(\omega>0)$

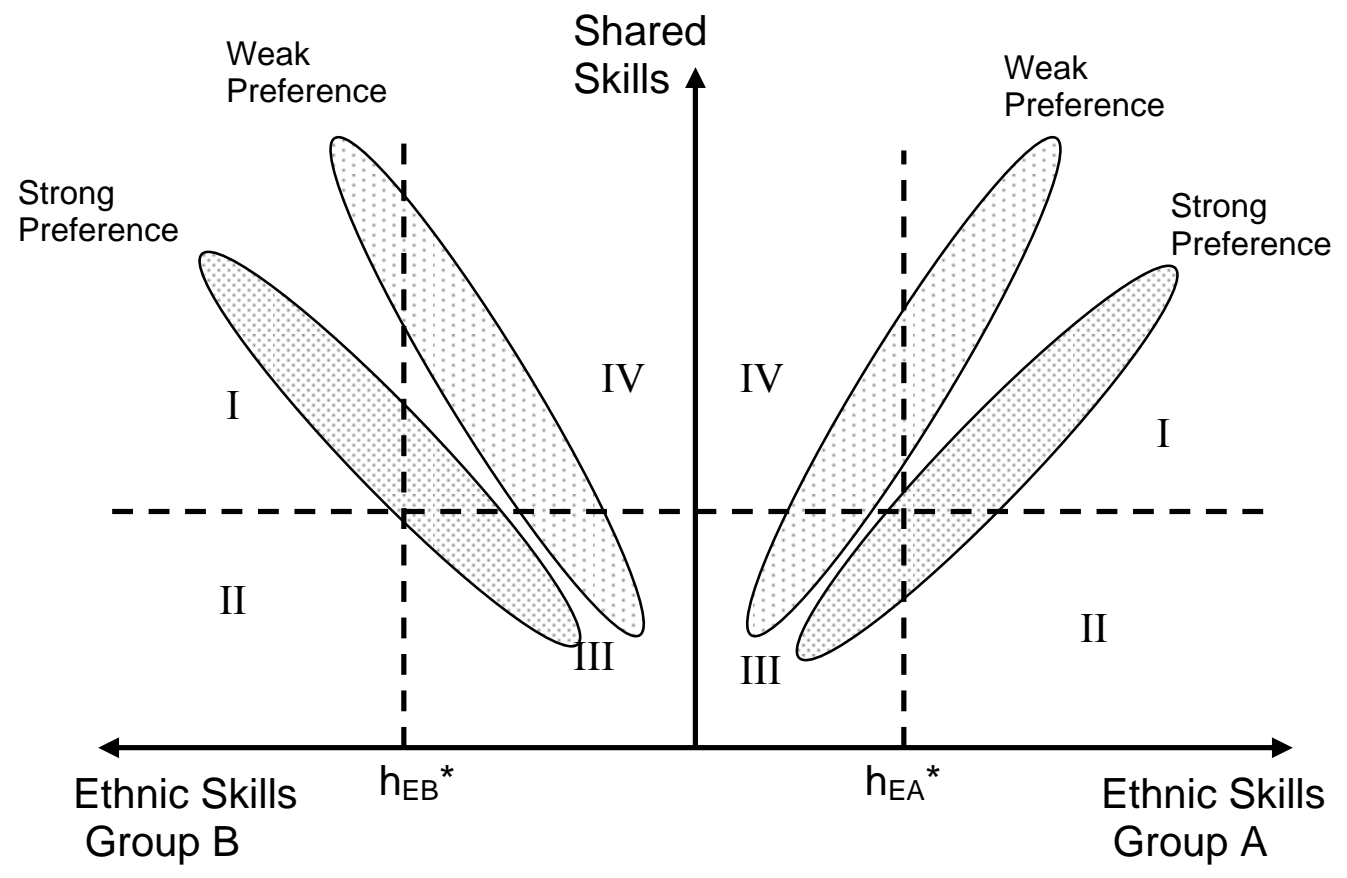


Figure $4 b$

Two Ethnic Groups with Negative Externalities $(\omega<0)$

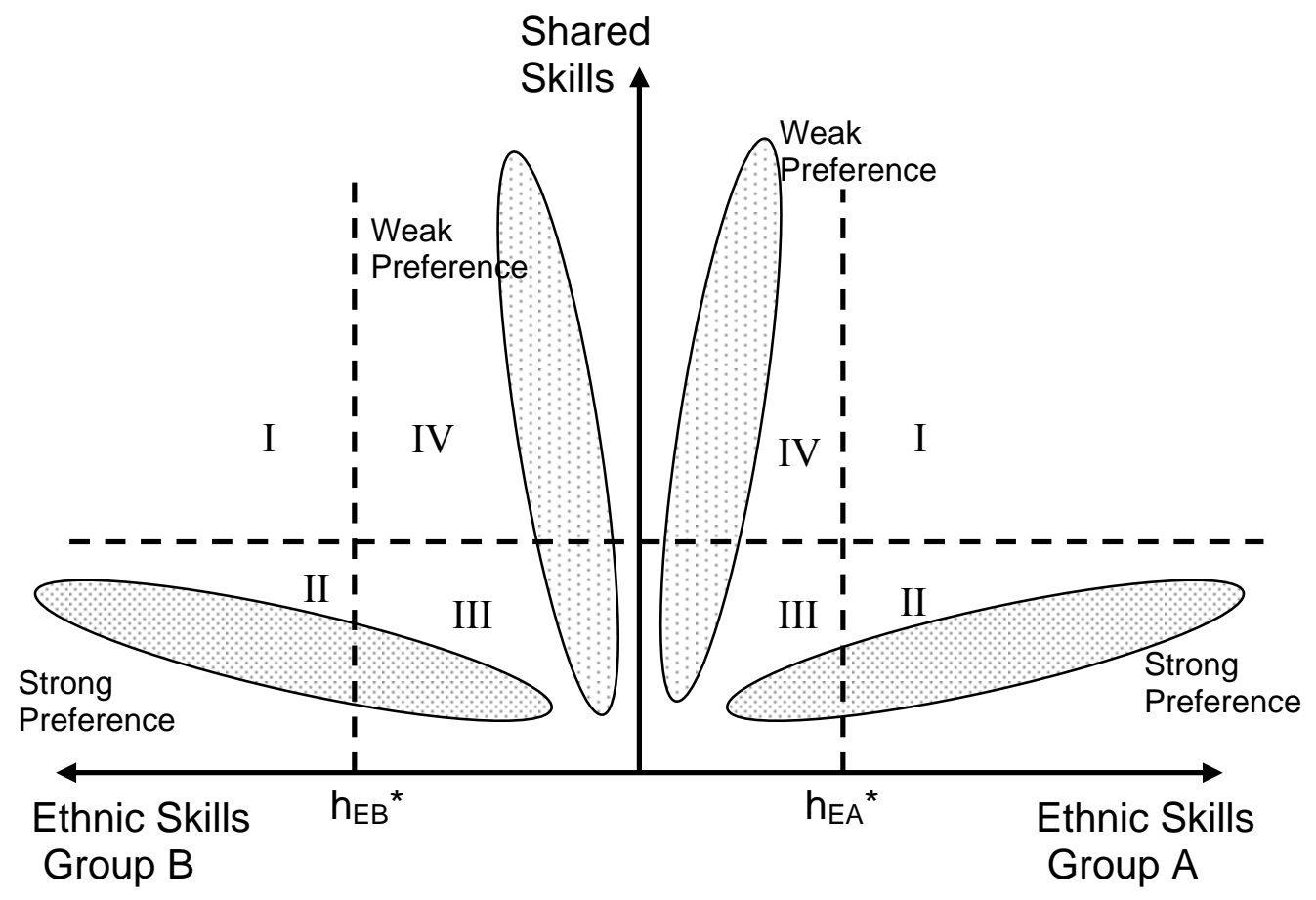

